\title{
Towards fast, reliable, and manufacturable DEAs: miniaturized motor and Rupert the rolling robot
}

Samuel Rosset and Herbert R. Shea

" Towards fast, reliable, and manufacturable DEAs: miniaturized motor and Rupert the rolling robot ", Proc. SPIE 9430, Electroactive Polymer Actuators and Devices (EAPAD) 2015, 943009 (April 1, 2015); doi:10.1117/12.2085279; http://dx.doi.org/10.1117/12.2085279

Copyright 2015 Society of Photo-Optical Instrumentation Engineers. One print or electronic copy may be made for personal use only. Systematic electronic or print reproduction and distribution, duplication of any material in this paper for a fee or for commercial purposes, or modification of the content of the paper are prohibited. 


\title{
Towards fast, reliable, and manufacturable DEAs: miniaturized motor and Rupert the rolling robot
}

\author{
Samuel Rosset, Herbert Shea \\ Ecole polytechnique fédérale de Lausanne, Switzerland
}

\begin{abstract}
Dielectric elastomer transducers (DETs) are known for their large strains, low mass and high compliance, making them very attractive for a broad range of applications, from soft robotics to tuneable optics, or energy harvesting. However, 15 years after the first major paper in the field, commercial applications of the technology are still scarce, owing to high driving voltages, short lifetimes, slow response speed, viscoelastic drift, and no optimal solution for the compliant electrodes. At the EPFL's Microsystems for Space Technologies laboratory, we have been working on the miniaturization and manufacturability of DETs for the past 10 years. In the frame of this talk, we present our fabrication processes for high quality thin-film silicone membranes, and for patterning compliant electrodes on the sub mm-scale. We use either implantation of gold nano-clusters through a mask, or pad-printing of conductive rubber to precisely shape the electrodes on the dielectric membrane. Our electrodes are compliant, time stable and present strong adhesion to the membrane. The combination of low mechanicalloss elastomers with robust and precisely-defined electrodes allows for the fabrication of very fast actuators that exhibit a long lifetime. We present different applications of our DET fabrication process, such as a soft tuneable lens with a settling time smaller than 175 microseconds, a motor spinning at $1500 \mathrm{rpm}$, and a self-commutating rolling robot.
\end{abstract}

Keywords: Dielectric elastomer actuators, fabrication process, manufacturability

\section{INTRODUCTION}

When SRI international published the first results on dielectric elastomer actuators over 15 years ago, it was clear that the main advantage of these new devices - compared to more traditional actuation methods - was the very large actuation strain that they can deliver (more than $100 \%$ area strain). ${ }^{1,2}$ With increasing understanding of the combined action of the Maxwell stress and the hyperelastic behaviour of the elastomer membrane, ${ }^{3}$ giant deformations up to $1692 \%$ were later demonstrated. ${ }^{4}$ In addition to their unmatched strain capabilities, DEAs are compliant and stretchable, lightweight, and energy efficient. However, despite their many interesting characteristics, DEAs have not yet been widely used by the industry, with only a few products based on this technology developed up to now. Some of the factors that prevents a wider use of DEAs in commercial products include high driving voltages, short lifetime, slow response speed coupled with viscoelastic creep, and the lack of a robust and reliable solution for the compliant electrodes.

The main focus of the microsystems for space technologies laboratory's research is to address some of the limitations mentioned above and develop robust and manufacturable DEAs exhibiting long lifetime (above 100 million cycles). Most of our research effort has been concentrated on compliant electrodes with the development of methods allowing the reproducible fabrication of electrodes that can be precisely patterned (resolution $<1 \mathrm{~mm}$ ) on thin elastomeric membranes. The following sections present our different fabrication processes for elastomer membranes and electrodes, followed by a few selected applications.

contact author: samuel.rosset@a3.epfl.ch 


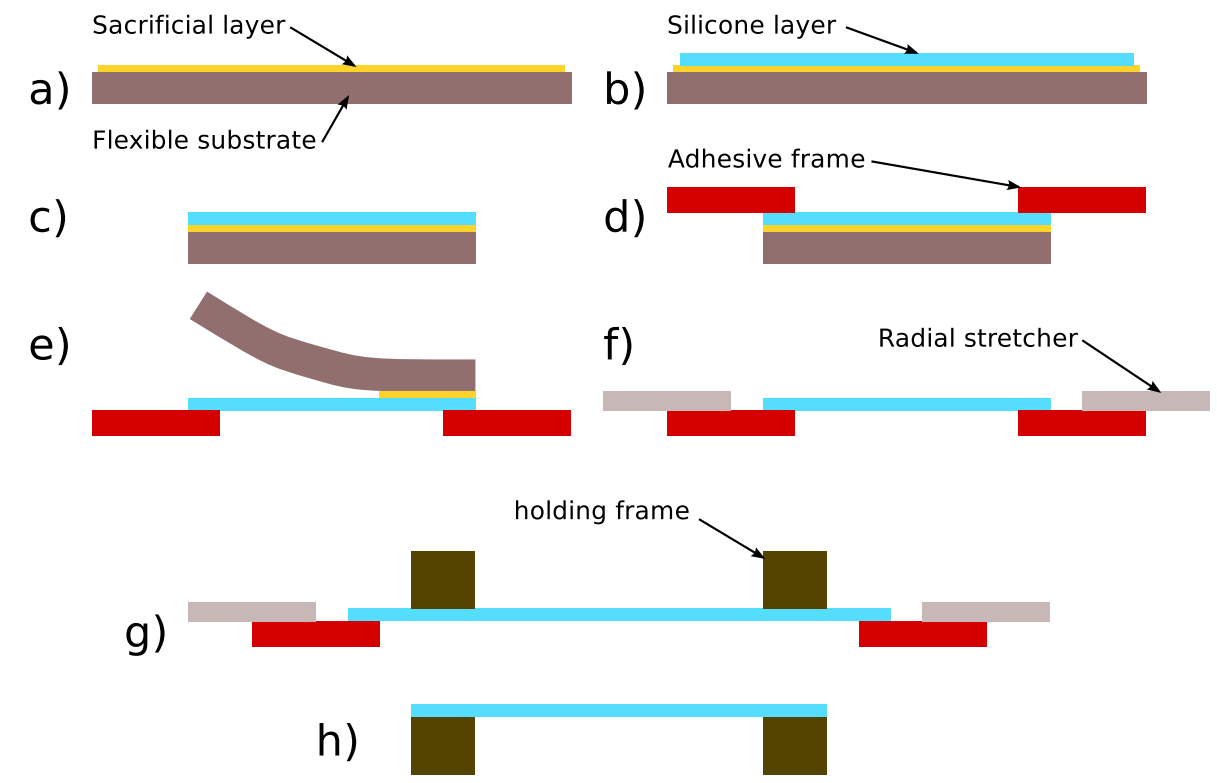

Figure 1. Silicone membrane fabrication process. a) Coating of a flexible substrate with a water-soluble sacrificial layer. b) Coating of the silicone layer on top of the dry sacrificial layer and cross linking in an oven. c) Cutting the large area membrane into circular sub-units. d) Application of an adhesive frame on the silicone membrane. e) Dissolution of the sacrificial layer in water and removal of the flexible substrate. f) Application of the adhesive frame on a radial prestretcher. g) The membrane is prestretched, and an adhesive-covered ring is applied on the stretched membrane. h) Cutting the membranes around the ring allows to finally obtain a stretched membrane fixed on a frame.

\section{FABRICATION PROCESSES}

\subsection{Elastomer membranes}

Different classes of elastomers can be used as membranes for DEAs, the three main ones being acrylic elastomers, silicones and polyurethanes. ${ }^{5}$ Acrylic elastomers, in the form of the proprietary adhesive foil VHB from $3 \mathrm{M}$, is the most widely used material in research labs, mainly because actuators based on this material exhibit very large strains. In addition, VHB is sold in film form and can be readily used, without the need to first fabricate thin and high-quality membranes. However, VHB suffers from a number of drawbacks that makes it unsuitable for industrial applications: due to its high mechanical loss tangent, the material reacts slowly to a force input, resulting in slow devices (full actuation strain is observed for driving frequencies below $0.1 \mathrm{~Hz}$ ). Viscoelastic creep also causes the strain to keep increasing with time under a constant applied voltage, making precise applications difficult without implementing a feedback mechanism. Finally VHB membranes are traditionally highly prestretched (stretch ratio typically between 3 and 4 in both in-plane directions), which makes them susceptible to stress relaxation $^{6}$ and/or membrane failure by tear propagation. ${ }^{7}$

Silicone elastomers present many advantages over VHB: they have a much lower mechanical loss factor that leads to much faster actuators (up to 3 orders of magnitude faster ${ }^{8}$ ), they do not need to be prestretched as much as VHB, reducing the risk of membrane rupture, and providing longer lifetime. Silicones are available in a large range of hardnesses, allowing to tailor the membrane to the application (a soft membrane to promote actuation strain, or a stiffer membrane to enhance the actuation force). Silicones being generally stiffer than VHB and having a lower relative permittivity, silicone-based actuators exhibit lower strain than similar actuators made with VHB. However, the actuation strain of VHB decreases rapidly with frequency because of its high viscous losses, and at driving frequencies above $1 \mathrm{~Hz}$, the actuation strain of silicone-based actuators exceeds that of VHB-based actuators. ${ }^{9}$ Consequently, except for demonstrating giant actuation strain at low frequencies, silicone-based actuators exhibit better performance, especially regarding stability in time, and we therefore systematically use silicone elastomers for our DEAs. 
Unlike VHB, silicone is usually not sold in membrane form, but as liquid components, and it is necessary to cast membranes in order to manufacture DEAs. The need to manufacture membranes is probably one of the reasons why VHB is still the dominating material used in the field of DEAs. We present here a simple process flow that allows manufacturing high-quality and large area silicone membranes for DEA applications (figure 1). The casting process should be done on a flexible high-quality defect-free substrate with a low surface roughness, as any defect from the substrate will be replicated in the membrane, which can lead to localized defects possibly causing premature breakdown. We use PET sheets (Melinex ST-506, DuPont Teijin films) as casting substrates. We then coat the substrate of A4 size with a water-soluble sacrificial layer (Dextran, poly(acrylic acid), or polyvinyl alcohol) ${ }^{10}$ with a wire bar applicator (figure $1 \mathrm{a}$ ). The dry thickness of the sacrificial layer coating is typically below $1 \mu \mathrm{m}$. Once the sacrificial layer is dry, the silicone layer is coated on top of it. The two components of the silicone elastomer are mixed together with a non-polar solvent (typically 2,2,4-trimethylpentane) with a planetary mixer in order to avoid the inclusion of bubbles. The purpose of the solvent is to reduce the viscosity of the mixture and make it suitable for casting. The quantity varies depending on the viscosity of the silicone, but is typically between $20 \%$ and $40 \%$ of the total mass. The mixture is cast using a variable gap applicator (ZUA 2000, universal applicator from Zehntner) on an automatic film coater (ZAA 2300, Zehntner) equipped with a vacuum plate holding the substrate in place (figure $1 \mathrm{~b}$ ). After casting the membrane, the layer is placed in an oven for 30 minutes at $80^{\circ} \mathrm{C}$ for the cross-linking process. The large (A4) silicone membrane is then cut into small circles $(50 \mathrm{~mm}$ diameter) for the prestretching process (figure $1 \mathrm{c}$ ). An annular adhesive frame is fixed on the membrane, the silicone layer being in contact with the adhesive (figure $1 \mathrm{~d}$ ). The assembly is dipped into a warm water bath, to dissolve of the sacrificial layer. The combination of the adhesive frame and the sacrificial layer allows to gently remove the flexible substrate without deforming the thin and soft silicone membrane (figure $1 \mathrm{e}$ ). In the absence of a sacrificial layer, it is necessary to pull on the membrane to release it from the casting substrate. Depending on the thickness of the membrane and its adhesion to the substrate, substantial stretching of the film may be required for the release, potentially leading to anisotropic mechanical properties in the film due to the Mullins effect. ${ }^{11}$ The impact of the Mullins effect on thin silicone membranes used for DEAs is described in more detailed in one of our previous articles. ${ }^{12}$

If prestretching is required for the application, the adhesive frame holding the released thin silicone membrane is fixed on a radial stretcher (figure $1 \mathrm{f}$ ). The the membrane is stretched equi-biaxially, and a plastic frame covered with silicone adhesive is applied on the stretched membrane (figure $1 \mathrm{~g}$ ). Finally, the excess membrane is cut around the frame, leaving the stretched membrane attached to the plastic frame (figure $1 \mathrm{~h}$ ).

\subsection{Compliant electrodes}

Compliant electrodes are one of the key components of DEAs, but are often overlooked and taken for granted. However, they play a key part in the performance of a DEA, influencing the strain, the response speed, and the lifetime of devices. There are many different methods used to make flexible and stretchable electrodes for DEAs, ${ }^{13}$ but hand-applied loose carbon black powder or carbon grease are the most widely used methods because, when used in combination with VHB membranes, they allow to quickly assemble a DEA demonstrator. However, their time stability is poor, and the hand application method restricts their usage to fairly large area devices and to the fabrication of lab prototypes.

We have investigated different methods to make compliant electrodes for DEA, which combine time stability, long lifetime and patternability on a small-scale $(<1 \mathrm{~mm})$. These three points are key parameters for the reproducible fabrication of small-size and robust DEAs that could be integrated in commercial products.

\subsubsection{Gold ion implantation}

We have used low energy gold ion implantation to create highly conductive robust compliant electrodes for DEAs. ${ }^{14}$ Filtered cathodic vacuum arc was used to implant gold ions into silicone membranes with an energy between $2.5 \mathrm{keV}$ and $5 \mathrm{keV}$, leading to a penetration of the ions of about $50 \mathrm{~nm}$ below the surface of the membrane. The implanted gold forms nano-clusters embedded into the membrane (figure 2). These nanoclusters touch each other without being mechanically attached, thus providing electrical conduction without stiffening the structure as a continuous metal film would. When the implanted layer is stretched, the clusters move relative to each other, thus maintaining contact and therefore electrical conduction. Depending on the quantity of ions, we were able to show conductivity up to uniaxial deformations of $175 \% .^{14}$ 

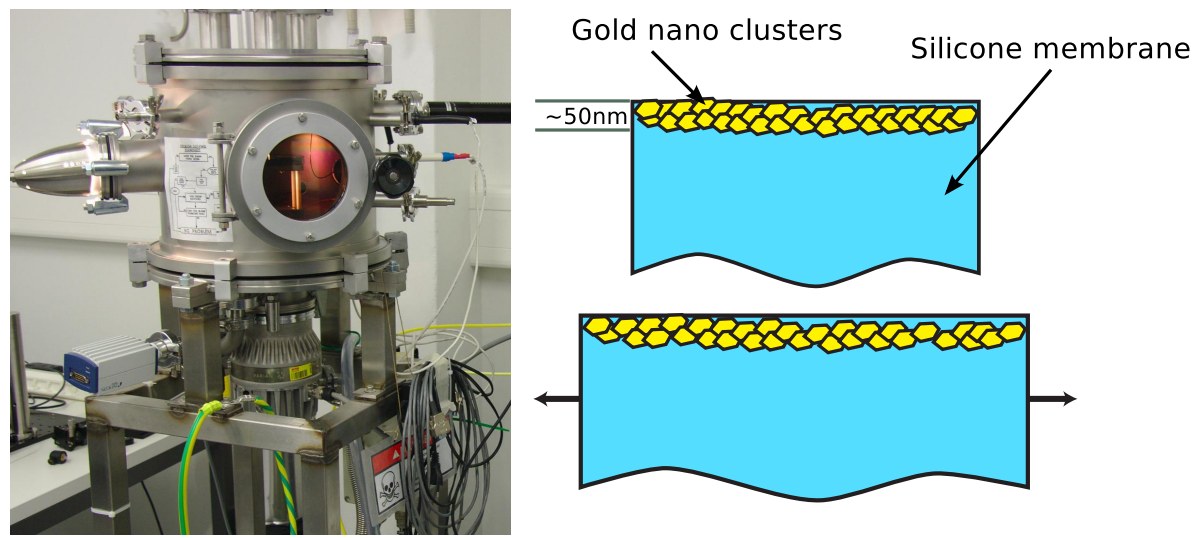

Figure 2. Left: filtered vacuum arc implanter for the fabrication of compliant electrodes at EPFL-LMTS. Right: gold ion implantation creates nano cluster in the first tens of nanometer below the surface of the silicone membrane. Upon deformation, the clusters can move relative to each other, thus maintaining a conductive path.

Gold ion implantation leads to highly conductive electrodes (surface conductivity in the range of a few hundreds Ohm to $1 \mathrm{k} \Omega$ per square) that present excellent adhesion to the surface, as the conductive particles are embedded into the elastomer. The electrodes can be precisely patterned using a shadow mask, or through a lift-off process, in order to only selectively expose the surface of the elastomer to the ion beam. Gold ion implantation causes a relatively small stiffening of the actuator structure compared to most of other metal-based approaches and does not require extra production steps, such as meander patterning or a corrugated substrate. We have applied gold ion implantation to many DEA applications, such as diaphragm actuators, ${ }^{15}$ miniaturized cell stretchers ${ }^{16}$ or zipping micropumps. ${ }^{17}$

Despite its many interesting properties, gold implanted electrodes have the drawback of being long to make, as the implantations are made in a vacuum chamber, which requires time to pump down. It also requires a quite unconventional equipment, which makes this technique difficult to apply in laboratories not equipped for filtered vacuum arc deposition.

\subsubsection{Conductive carbon black/silicone composite}

As an alternative to gold ion implantation, we have worked on a faster method to produce compliant electrodes, more adapted to a growing number of lab users requiring to apply electrodes on elastomer membranes. Carbonbased electrodes are generally interesting because of their low impact on the stiffness of the device and their ease of application. ${ }^{13}$ Their relatively low conductivity is compensated by the fact that they can sustain very large strains while remaining conductive, and that DEAs being electrostatic devices, they usually don't require very conductive electrodes. Consequently, we decided to investigate carbon-based electrodes. However, in addition to the basic properties of electrical conductivity and compliance, there are a few key requirements that we wanted our new method to meet, so as to present a viable route towards industry-compatible compliant electrodes:

- Possibility to pattern the electrodes on a small scale $(<1 \mathrm{~mm})$.

- Short time required to apply the electrode.

- Reproducible electrode geometry (shape and thickness) and electrical properties

- Homogeneous electrode thickness

- Good adhesion of the electrode to the silicone membrane

- Resistance to abrasion and wear (the electrode can be touched without being damaged)

- Time stability of the electrode (resistance stable in time and with the number of stretching cycles) 


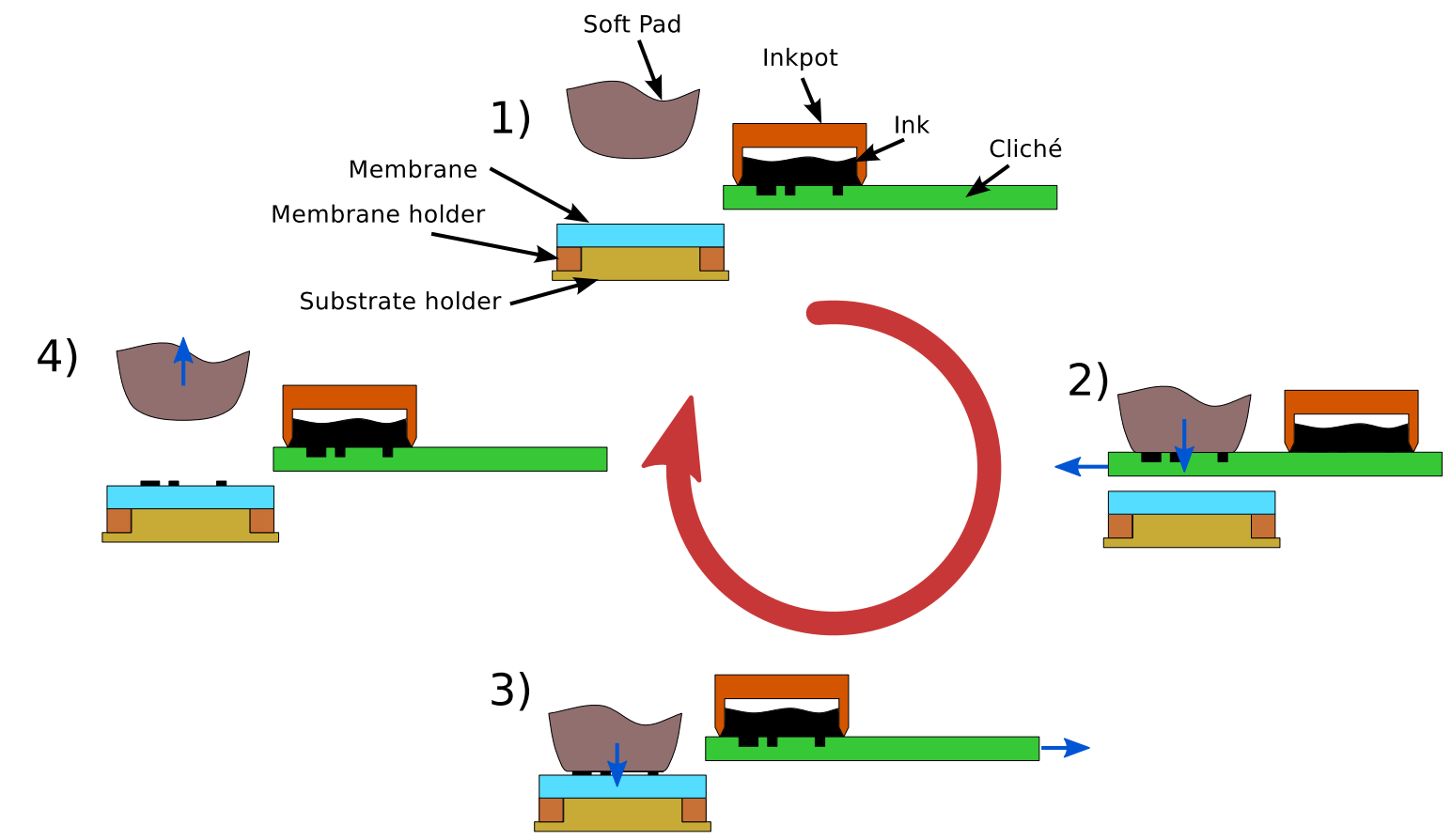

Figure 3. Schematic representation of a pad printing cycle: 1) A cliché (steel plate in which the design to be transferred is etched) is installed in the machine and covered by an inkpot filled with the solution to be printed. 2) The cliché is shifted on the left to present the cavities filled with ink to a smooth stamp, which is moved downwards to pick up the ink from the cliché. 3) The cliché is retracted below the inkcup to refill the structures with ink. At the same time, the stamp is lowered to the membrane in order to transfer the ink from the pad to the surface of the membrane. 4) The stamp moves back upwards.

In view of the previous requirements, loose carbon powder and carbon grease, although widely used for DEAs are not suitable candidates, as they are susceptible to abrasion and wear and do not adhere well to the membrane. We therefore use carbon black mixed into a cross-linked silicone matrix coupled with a stamping process in order to pattern precisely defined electrodes that present a high adhesion to the membrane and a good resistance to abrasion, thus leading to very resilient electrodes. Among the different possible patterning techniques that are compatible with a mixture containing silicone and carbon black particles, we have selected pad printing, because although it is a contact method, only a soft and smooth pad comes into contact with the membrane. This avoids any risk of deforming, indenting or damaging the membrane, as can be possible when hard masks or meshes come into contact with the membrane, such as with screen printing. The ideal way of patterning electrodes on a soft suspended membrane while avoiding deformation and damages is a non-contact method, such as inkjet printing, but it often requires a lot of ink development to obtain a jettable solution. With pad printing, many different solutions can be patterned, encompassing a large range of viscosities and surface tensions. The presence of agglomerates is not problematic.

The pad printing process is described on figure 3. The design to be transferred on the membrane is etched into a steel plate to a depth of about $20 \mu \mathrm{m}$. An inkpot containing the solution to be printed is placed on the etched steel plate (called a cliché), the lip of the inkpot acting as a doctor blade. At the beginning of the cycle, the inkpot covers the part of the cliché where the design is etched (figure 3 step 1 ). In a second step (figure 3 step 2), the cliché is moved to the left, positioning the etched cavities filled with ink below a soft stamp. The stamp is lowered on the cliché to pick-up the ink. Then (figure 3 step 3 ), the cliché retracts allowing the etched structures to return below the inkcup to be filled with ink. The stamp can then move downwards to apply the patterned ink on the membrane, which is placed on a substrate holder that can be precisely positioned. In a final step (figure 3 step 4), the pad is removed from the membrane, leaving the ink on the surface of the membrane. 


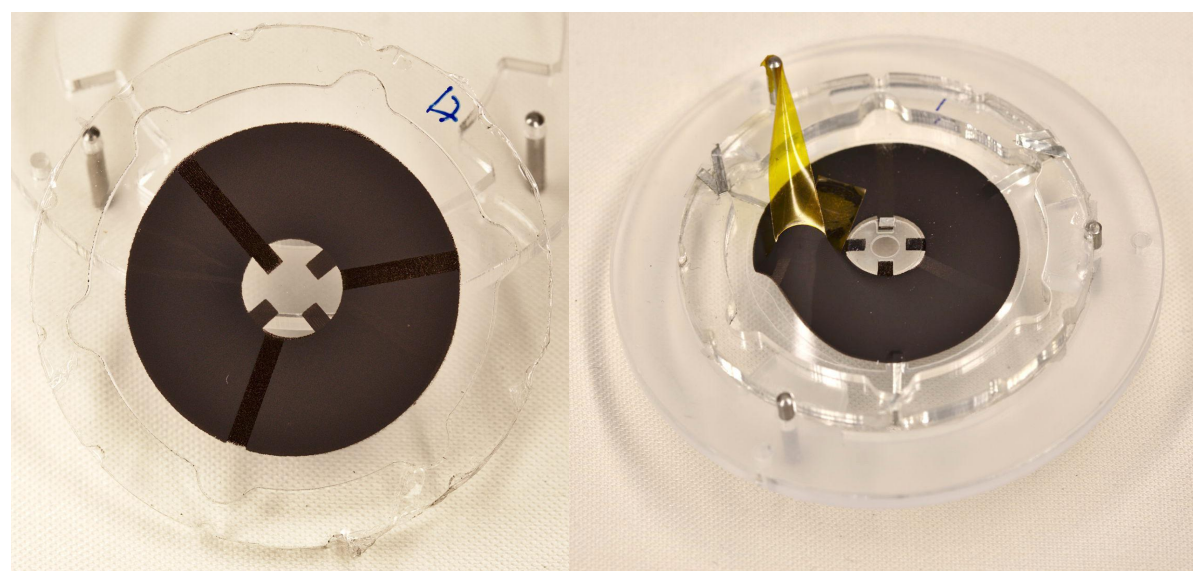

Figure 4. Left: carbon black and composite electrodes patterned by pad printing on both sides of a silicone membrane. Right: adhesion of the cured electrode on the membrane is demonstrated by a scotch tape test.

A new membrane can then be positioned on the substrate holder, and a new printing cycle can start. It only takes a few seconds to pattern an electrode on the membrane. After application, the electrode must be cured in an oven at $80^{\circ} \mathrm{C}$ for 30 minutes to allow the silicone in the ink to crosslink. However, many electrodes can be printed and then batch cured in the oven, thus making pad printing a very fast and efficient technique for the series production of DEAs.

An example of pad printed electrode is shown on figure 4 left. The top-side of the membrane is patterned with 3 independent electrodes, and there is a ground electrode on the backside of the membrane. Thanks to its silicone matrix, the ink presents a very good adhesion to silicone membrane, as shown in figure 4 right, where a tape with silicone adhesive is applied on a cured electrode. As can been seen in the picture, pulling on the tape does not damage the electrode nor de-laminate it from the elastomer membrane: pad-printing of carbon black/silicone composite leads to very sturdy and robust electrodes.

\section{SELECTED APPLICATIONS}

The combination of high-quality silicone membranes produced with the process described in section 2.1 with patternable compliant electrodes applied by pad-printing (section 2.2.2), leads to the reproducible fabrication of reliable and robust miniaturized DEAs that combine fast response speed, absence of drift and long lifetime. The following sections gives a few selected examples of actuators that have been developed in our laboratory.

\subsection{Ultra-fast soft tuneable lens}

Because of their large actuation strain, DEAs are interesting candidates for soft tuneable optical devices, and different applications have been demonstrated, such as diffraction gratings, ${ }^{18,19}$ lenses $^{20,21}$ and phase retarders. ${ }^{22}$ Most of the aforementioned devices are based on acrylic elastomer (VHB) membranes, which allow them to demonstrate an extended tuning range. However, most real-world applications require not only a large tuning range, but the ability to quickly change configuration, and to hold a stable position during a given time. Because of its high viscous losses and viscoelastic creep, VHB does not offer a viable solution without using some sort of feedback mechanism such as self-sensing. ${ }^{23}$

To show the potential gain between two geometrically identical devices but using different materials, we have worked in collaboration with Federico Carpi to manufacture a bio-inspired soft tuneable lens ${ }^{20}$ both with VHB and silicone elastomer. The lens consists of two membranes bonded together encapsulating a small amount of optical fluid at the centre of the membrane, thus forming a lens. An annular electrode is patterned around the lens (figure 5). When the device is activated, the expansion of the electrode compresses the lens and modifies its radius of curvature, and hence its focal length. Both the static focal length variation induced by the electric field, and the response speed of the lenses were characterized. ${ }^{8}$ 

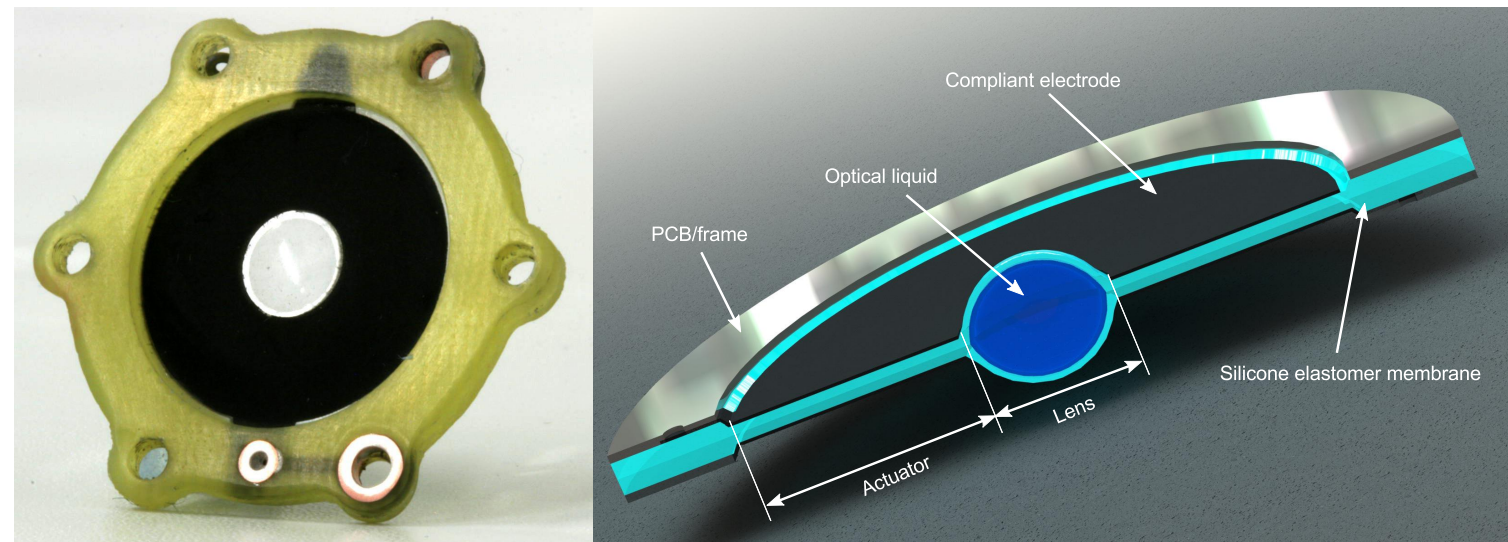

Figure 5. Left: Picture of the assembled soft tuneable lens. Right: Lens cross-section. Two membranes are bonded together, with a small amount of liquid encapsulated at the center to form a lens. An annular electrode is patterned around the lens. When the device is activated, the electrode expansion compresses the lens, resulting in a change of curvature, and a voltage-tuneable focal length. The measured settling time of the silicone-based lens was less than $200 \mu \mathrm{s}^{8}$
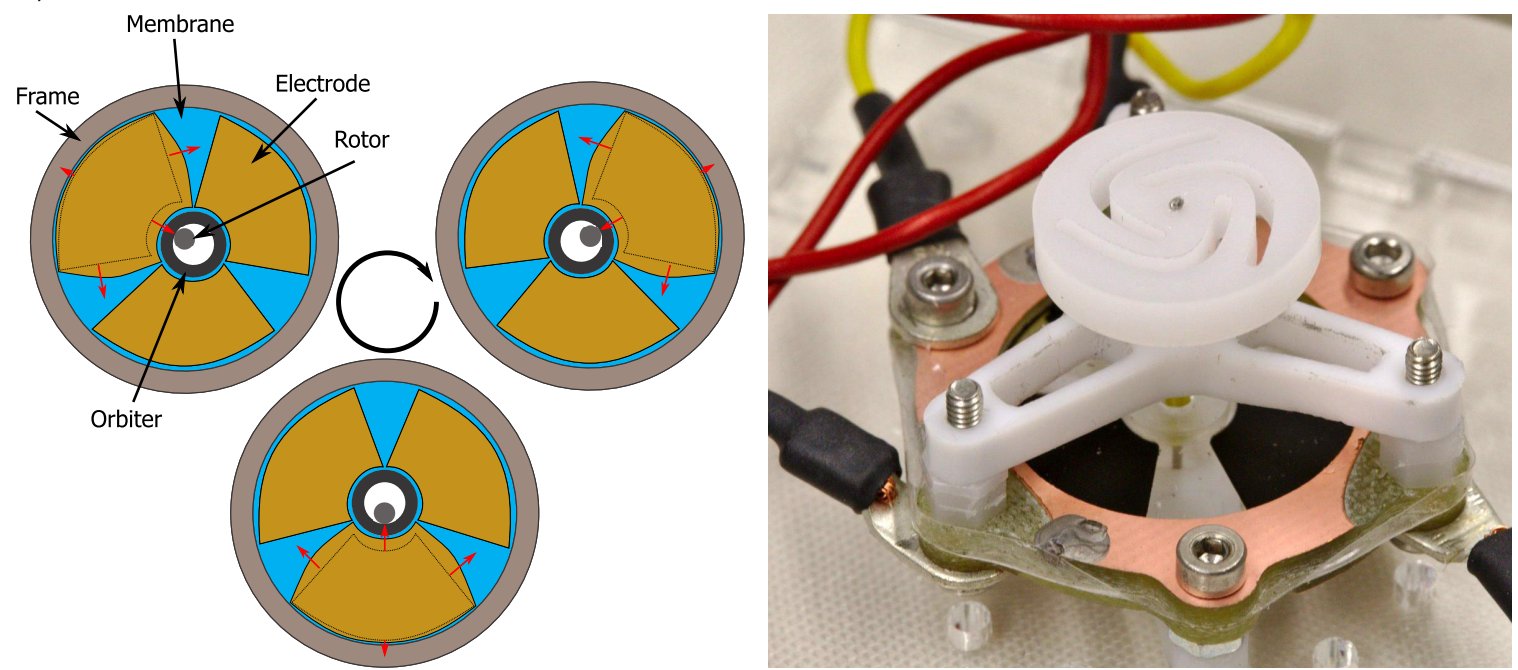

Figure 6. Left: Working principle of the elastomeric motor. 3 independent electrodes are patterned on the membrane. The electrodes are sequentially activated, causing the displacement of an annular orbiter placed at the centre of the membrane. The motion of the orbiter causes the central shaft to rotate due to friction. Right: Picture of the assembled motor. The diameter of the membrane is $20 \mathrm{~mm}$.

The characterization of the silicone-based tunable lenses shows that they are able to exhibit a static change of focal length of up to $25 \%$ of the original value, whereas VHB lenses can be tuned up to $37 \%$ of their initial focal length. ${ }^{8}$ However, the silicone lenses exhibit a very fast response speed to a voltage step input, with a settling time of less than $200 \mu \mathrm{s}$. Its frequency response is flat up to more than $1 \mathrm{kHz}$, which represents a bandwidth 3 orders of magnitude larger compared to a VHB lens of equal geometry. ${ }^{8}$ In addition to a very fast response speed, the lens can be actuated for more than 400 million cycles without degradation in response amplitude. This shows that by selecting adequate materials and fabrication techniques, DEAs can combine softness and compliance with high response speed and long lifetimes. 


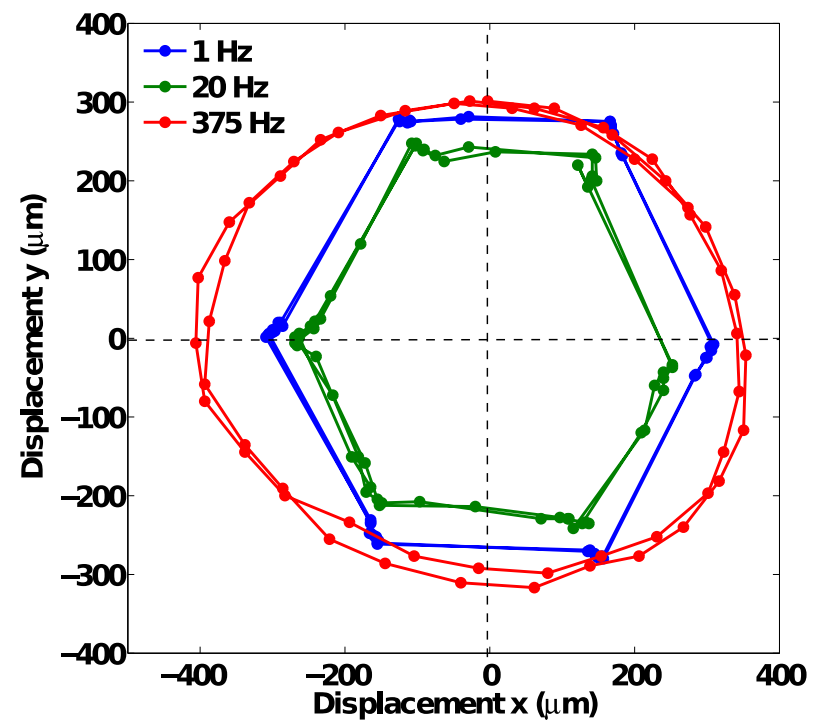

Figure 7. In-plane displacement of the centre of the orbiter, measured with a high speed camera at $80 \mathrm{~V} / \mu \mathrm{m}$ and for different frequencies. At the in-plane resonance frequency of the structure $(375 \mathrm{~Hz})$, the hexagonal pattern is transformed into a circular-like motion of larger amplitude.

\subsection{DEA motor}

The combination of silicone membranes with pad-printed electrodes for the realisation of small-size fast and robust DEAs has also been applied to the soft rotary motor developed by Anderson et al. ${ }^{24}$ In collaboration with the biomimetics lab from the university of Auckland, we have manufactured a miniaturized version of the rotary elastomeric motor (figure 6): three electrically-independent electrodes are patterned by pad printing on a $20 \mathrm{~mm}$-diameter prestretched silicone membrane. At the centre of the membrane lies an annular orbiter with a shaft going through it. The electrode are successively activated, which causes the orbiter to translate in a circular-like motion around the shaft, making the later turn through friction.

The motor is driven by a high voltage electronic circuit that delivers three square signals with a duty cycle of $50 \%$, and a $120^{\circ}$ phase shift between channels. A high speed camera is used to track the in-plane displacement of the orbiter for different frequencies of the driving signal (figure 7). The driving electric field is fixed at $80 \mathrm{~V} / \mu \mathrm{m}$. This characterization is made without the shaft (i.e. just the membrane with the orbiter fixed at the centre), in order to have an unobstructed field of view for the camera. At low driving frequency $(1 \mathrm{~Hz})$, the orbiter jolts from one position to the other at each of the 6 changes of electrode configuration during a cycle, thus forming an hexagonal-like pattern. At $20 \mathrm{~Hz}$, the hexagonal pattern is still clearly visible, with a slightly smaller amplitude. At the in-plane resonance frequency of the structure $(375 \mathrm{~Hz})$, a regular circle-like pattern is observed with a larger amplitude than the hexagon observed at low frequency. A shaft with a $0.6 \mathrm{~g}$ proof mass at the top is then placed through the orbiter to obtained the full motor shown on figure 6 right, and the rotation speed of the proof mass is monitored with the help of a high speed camera. The proof mass was observed to rotate steadily at $1500 \mathrm{rpm}(25 \mathrm{rev} / \mathrm{s})$. The motor pictured on figure 6 right has been assembled for the EAP in-action session of EAPAD 2013 where it was demonstrated continuously for more than 1 hour. 2 years later, this device is still working perfectly and is regularly shown to visitors and during exhibitions. It has worked many hours and accumulated several tens of million of cycles without any performance degradation. A video of the elastomer rotary motor can be found on our website: http://lmts.epfl.ch/EAPmotor

\subsection{Rupert the rolling robot}

We have developed the motor concept further by fabricating a self-commutating rolling robot scooting along a circular track (figure 8 and 9). Rupert is composed of two membranes (to increase the output force), each having three independent electrodes patterned on their surface. A rotation axis is mounted through the center of the 

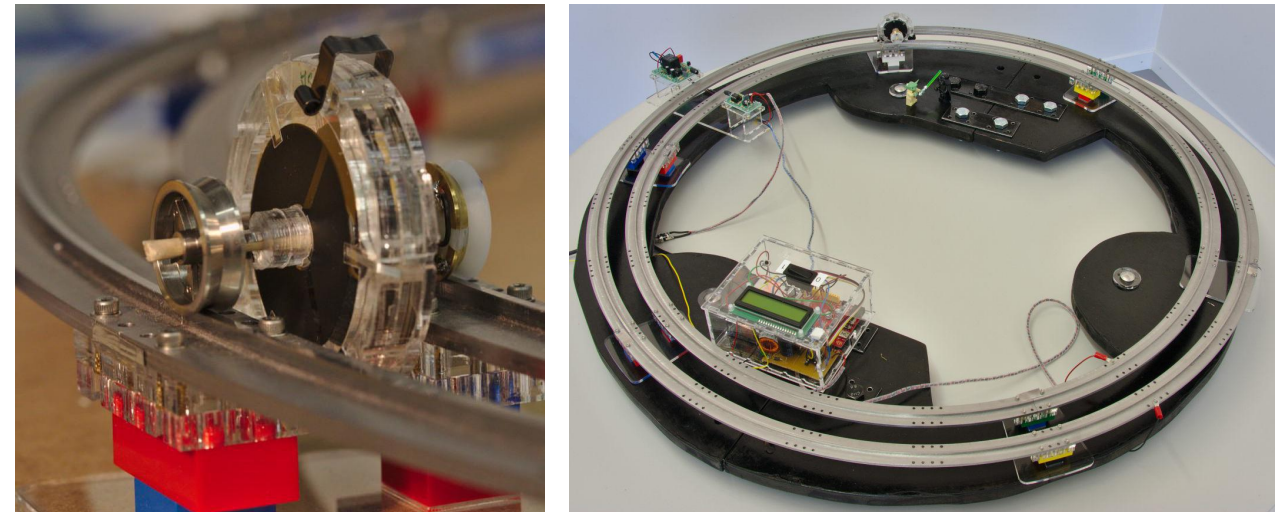

Figure 8. Left: close-up view of Rupert, the self-commutating rolling robot. Right: Rupert's track with a mean perimeter of $2.15 \mathrm{~m}$. It is equipped with an infrared light gate in order to track Rupert's travelled distance and speed of each turn.

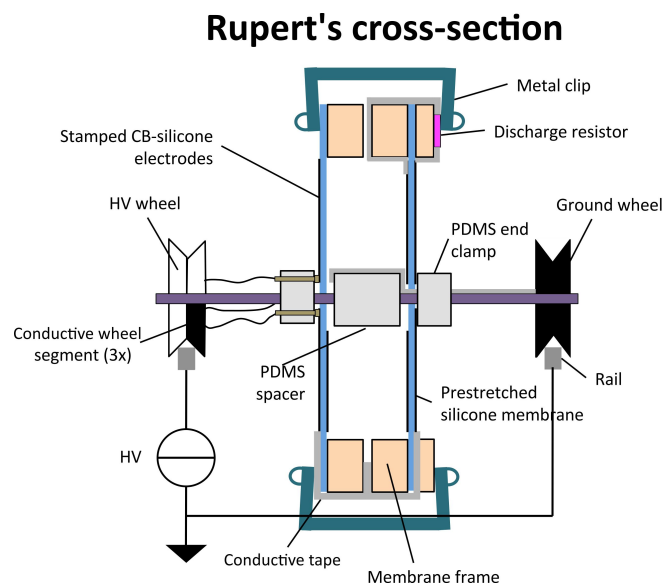

\section{STATIC}

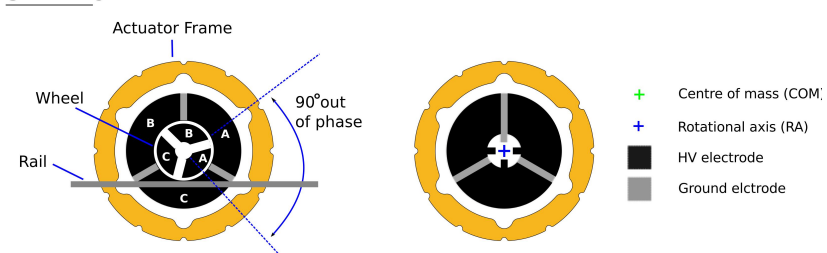

\section{ROLLING}
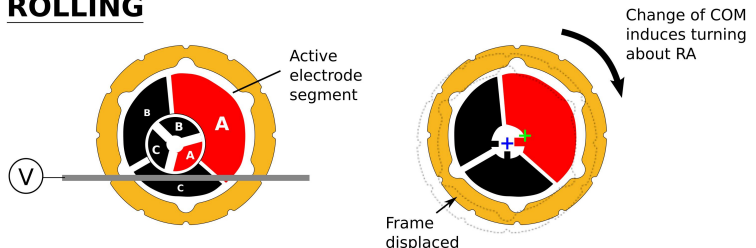

Figure 9. Left: Cross-section of the rolling robot The rails are used to power the robot. Right: Rupert's working principle. Each of the three electrodes is linked to a sector of the HV wheel. The activated electrode expands and causes a displacement of the outer frame of the robot compared to its centre of rotation, tipping the robot and making it roll. The rolling motion is sustained by subsequent sectors of the HV wheel coming into contact with the rail.
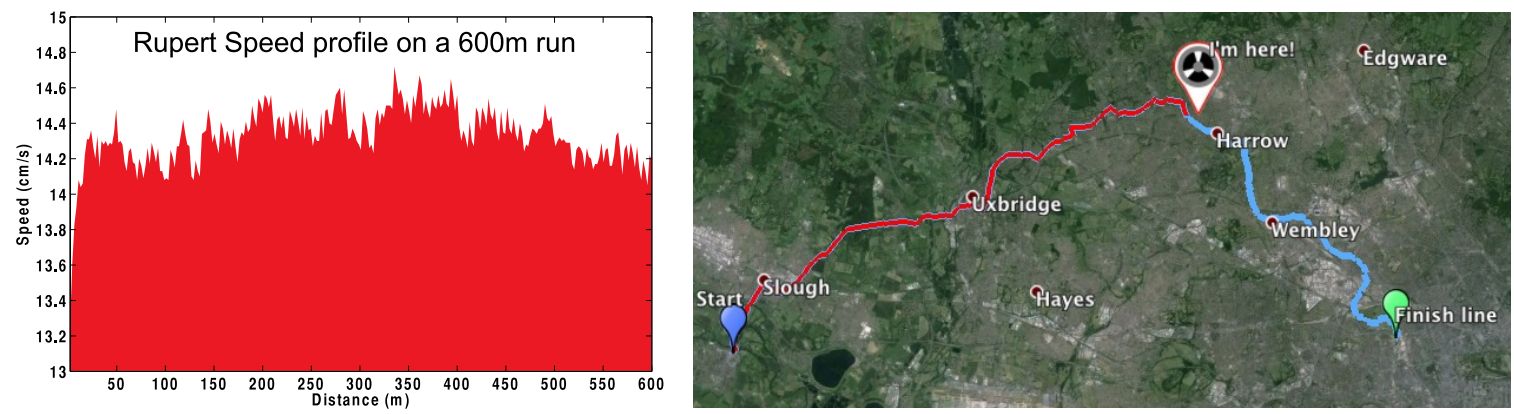

Figure 10. Left: Rupert's speed for each turn on a 600 m run. Right: Rupert's progression on the 1908 London Olympic games marathon before failure. Rupert travelled $25.8 \mathrm{~km}$ (61\% of the marathon distance). 
membranes and wheels are fixed on the extremities of the shaft. The track on which Rupert rolls is circular and made with metallic rails that provide the high voltage to the device. The wheel in contact with the high voltage rail is segmented into three conductive zones, each connected to one of the three electrodes. When one of the sectors of the high voltage wheel is in contact with the rail, the corresponding electrode expands. This causes the outer plastic frame of the robot to be displaced outwards and leads to a shift of the center of mass of the device compared to its axis of rotation, thus making the robot roll due to the generated torque. The imbalance is maintained during the rolling motion, as the successive sectors of the high voltage wheel come into contact with the rail. A discharge resistor is used to de-activate the electrodes that are in a floating state when their respective sector of the high voltage wheel is not in contact with the rail.

Rupert rolls steadily at a speed typically between $10-20 \mathrm{~cm} / \mathrm{s}$ which depends on the actuation voltage. Figure 10 shows Rupert's speed measured on each track turn with the infra-red light gate for a constant voltage applied to the rails. The objective is to have Rupert roll on its track over the distance of a marathon. A script maps the live progression of Rupert on the original marathon itinerary form the 1908 London Olympic games (figure 10). The tested unit was able to travel a distance of $25.8 \mathrm{~km}$ on the tracks before failing, which represents $61 \%$ of the total marathon distance, and 12001 turns on its track. The failure was caused by one of the electrical contacts on the electrodes which were made using conductive tape. One of the strips of tape lost adhesion to the electrode, which caused arcing between the tape and and the electrode, leading to the erosion of the electrode material. A more robust way of making the contact between the external circuit and the soft membrane, such as using a PCB with contact pads as is the case for the lens (figure 5) or the motor (figure 6) would solve the issue and allow the robot to easily travel the distance of a marathon.

The failure caused by the electrical contact between the stiff external conductors and the soft DEAs shows that reliability and lifetime is not only determined by the dielectric membrane and the compliant electrodes, but also by the interface to the external circuit providing the charges. The transition between the rigid frame holding the membrane and the soft elastomer is delicate, and must not be neglected for devices which must survive a large number of cycles. A video of Rupert the rolling robot can be found on our website: http://lmts.epfl.ch/rupert

\section{CONCLUSIONS}

Real-world applications of dielectric elastomer actuators require not only large output strains, but also the ability to be repeatedly actuated over a large number of cycles without noticeable performance degradation, and to do so at a reasonable speed (at least several $\mathrm{Hz}$ ). In addition, actuators must be produced reproducibly and efficiently. Here, we have shown how the combination of silicone elastomer membranes and high quality patterned compliant electrodes leads to the reproducible manufacture of miniaturized and fast DEAs that present a very long lifetime. Some actuators fabricated more than 2 years ago are still in perfect working condition, thanks to the very stable electrodes, and cyclic actuation of more than 400 million cycles have been demonstrated in the case of the tuneable lens. In addition to the high driving voltages (which are not addressed in this contribution), manufacturability, miniaturisation, response speed and lifetime are keypoints that need to be solved in order to see a broader use of DEAs in the industry. The examples given here (both fabrication processes and devices) show that soft actuators can be fast, small, and work over a large number of cycles.

\section{ACKNOWLEDGMENTS}

The authors wish to thank the lab members working on soft transducers for their precious contributions to the results presented here: Seun Araromi, Juan Zarate, Luc Maffli, Alexandre Poulin, Jun Shintake, Nadine Besse, Alexis Marette, Anna Kamolwat, and Samuel Schlatter. This work has been partially funded by the Swiss National Science Foundation grant 200020-153122.

\section{REFERENCES}

1. R. E. Pelrine, R. D. Kornbluh, and J. P. Joseph, "Electrostriction of polymer dielectrics with compliant electrodes as a means of actuation," Sensors and Actuators, A: Physical 64(1), pp. 77-85, 1998. 
2. R. Pelrine, R. Kornbluh, Q. Pei, and J. Joseph, "High-speed electrically actuated elastomers with strain greater than 100\%," Science 287(5454), pp. 836-839, 2000.

3. Z. Suo, "Theory of dielectric elastomers," Acta Mechanica Solida Sinica 23(6), pp. 549-578, 2010.

4. C. Keplinger, T. Li, R. Baumgartner, Z. Suo, and S. Bauer, "Harnessing snap-through instability in soft dielectrics to achieve giant voltage-triggered deformation," Soft Matter 8, pp. 285-288, 2012.

5. P. Brochu and Q. Pei, "Advances in dielectric elastomers for actuators and artificial muscles," Macromolecular Rapid Communications 31(1), pp. 10-36, 2010.

6. S. Michel, X. Zhang, C. Wissler, M.and Loewe, and G. Kovacs, "A comparison between silicone and acrylic elastomers as dielectric materials in electroactive polymer actuators," Polymer International 59(3), pp. 391399, 2010 .

7. P. Lochmatter and G. Kovacs, "Design and characterization of an active hinge segment based on soft dielectric eaps," Sensors and Actuators, A: Physical 141(2), pp. 577-587, 2008.

8. L. Maffli, S. Rosset, M. Ghilardi, F. Carpi, and H. Shea, "Ultrafast All-Polymer Electrically Tunable Silicone Lenses," Advanced Functional Materials, 2015.

9. M. Molberg, Y. Leterrier, C. J. G. Plummer, C. Walder, C. Loewe, D. M. Opris, F. A. Nuesch, S. Bauer, and J.-A. E. Manson, "Frequency dependent dielectric and mechanical behavior of elastomers for actuator applications," Journal of Applied Physics 106(5), p. 054112, 2009.

10. V. Linder, B. D. Gates, D. Ryan, B. A. Parviz, and G. M. Whitesides, "Water-soluble sacrificial layers for surface micromachining," Small 1, pp. 730-736, Jul 2005.

11. L. Mullins, "Softening of rubber by deformation," Rubber Chemistry and Technology 42(1), pp. 339-362, 1969 .

12. S. Rosset, L. Maffli, S. Houis, and H. Shea, "An instrument to obtain the correct biaxial hyperelastic parameters of silicones for accurate DEA modelling," in Proceedings of SPIE - The International Society for Optical Engineering, 9056, p. 90560M, 2014.

13. S. Rosset and H. Shea, "Flexible and stretchable electrodes for dielectric elastomer actuators," Applied Physics A: Materials Science \& Processing 110, pp. 281-307, 2013.

14. S. Rosset, M. Niklaus, P. Dubois, and H. Shea, "Metal ion implantation for the fabrication of stretchable electrodes on elastomers," Advanced Functional Materials 19(3), pp. 470-478, 2009.

15. S. Rosset, M. Niklaus, P. Dubois, and H. Shea, "Large-stroke dielectric elastomer actuators with ionimplanted electrodes," Journal of Microelectromechanical Systems 18(6), pp. 1300-1308, 2009.

16. S. Akbari and H. R. Shea, "An array of $100 \mathrm{um} x 100 \mathrm{um}$ dielectric elastomer actuators with $80 \%$ strain for tissue engineering applications," Sensors and Actuators A: Physical 186, pp. $236-241,2012$.

17. L. Maffli, B. O'Brien, S. Rosset, and H. Shea, "Pump it up," in Proceedings of SPIE - The International Society for Optical Engineering, Y. Bar-Cohen, ed., Proceedings of SPIE 8340, pp. 8340-2Q, 2012.

18. M. Kollosche, S. Doering, J. Stumpe, and G. Kofod, "Voltage-controlled compression for period tuning of optical surface relief gratings," Optics Letters 36, pp. 1389-1391, Apr 2011.

19. M. Aschwanden, D. Niederer, and A. Stemmer, "Tunable transmission gratings based on dielectric elastomer actuators," in Proceedings of SPIE - The International Society for Optical Engineering, 6927, pp. 6927-56, SPIE, (San Diego, California, USA), 2008.

20. F. Carpi, G. Frediani, S. Turco, and D. De Rossi, "Bioinspired tunable lens with muscle-like electroactive elastomers," Advanced Functional Materials 21(21), pp. 4152-4158, 2011.

21. S. Shian, R. M. Diebold, and D. R. Clarke, "Tunable lenses using transparent dielectric elastomer actuators," Opt. Express 21, pp. 8669-8676, Apr 2013.

22. M. Beck, R. Fiolka, and A. Stemmer, "Variable phase retarder made of a dielectric elastomer actuator," Optics Letters 34, pp. 803-805, Mar 2009.

23. S. Rosset, B. M. O’Brien, T. Gisby, D. Xu, H. R. Shea, and I. A. Anderson, "Self-sensing dielectric elastomer actuators in closed-loop operation," Smart Materials and Structures 22(10), p. 104018, 2013.

24. I. Anderson, T. Hale, T. Gisby, T. Inamura, T. McKay, B. O'Brien, S. Walbran, and E. Calius, "A thin membrane artificial muscle rotary motor," Applied Physics A: Materials Science and Processing 98(1), pp. 75-83, 2010. 